\title{
Consenso Nacional de Urgencias Endoscópicas en Pediatría 2016. Resumen ejecutivo
} Emergency Endoscopic Consensus 2016. Executive summary

Comité Nacional de Gastroenterología

Coordinación: Dra. Inés Ninomiya, Dra. Carolina Riga.

Comité Nacional de Gastroenterología: Dres. Jorge Villarruel, Fernando Vinuesa, Lucio González, Jorge Rubin, Federico Ussher, Gustavo Aliverti, María de los Ángeles Valdez Suárez, Valeria Taire, José Vásquez, Sofía Paz.

Revisión por Comité de Emergencia y Cuidados Críticos, de Medicina Interna, de Prevención de Lesiones, Asociación Argentina de Cirugía Pediátrica.

Dres. Tomás Iolster, Daniel Liberto, Andrés Villa de Villafañe, Leonardo De Lillio, Ingrid Waisman, Vanesa Zaslavsky, Salome Santarcangelo, Claudia Sosa, Gabriela Varone, Andrea Belardinelli y Marta Beatriz Caro.

http:/ /dx.doi.org/10.5546/aap.2017.96

Véase el texto completo en formato electrónico, en la sección "Consensos" del sitio web de la Sociedad Argentina de Pediatría www.sap.org.ar

El consenso consta de 3 secciones: Urgencias endoscópicas por Hemorragia digestiva, por Ingestión de cuerpos extraños e Ingestión de cáusticos. En cada una de ellas se expone una revisión actualizada, una guía de diagnóstico, algoritmos y terapéutica recomendada.

\section{HEMORRAGIA DIGESTIVA}

Hemorragia digestiva (HD) es la pérdida de sangre manifiesta u oculta, que se origina en cualquier segmento del tubo digestivo, primario o secundario a una enfermedad general.

En el $80 \%$ de los casos no tiene consecuencias graves y se autolimita, pero nunca debe ser minimizada. El papel del pediatra y gastroenterólogo es evaluar la magnitud de las pérdidas y las consecuencias hemodinámicas, identificar el origen y la etiología del sangrado e instaurar un tratamiento efectivo. La solución definitiva puede ser médica, endoscópica o quirúrgica.

La Endoscopia es el método de elección para evaluar el origen de la hemorragia digestiva alta. Lo ideal es realizarla en las primeras 24 horas del inicio del evento, en las que se identifica el origen del sangrado en el 90 - 95\% de las lesiones; si se realiza 72 horas después es del $48 \%$. En pediatría, por lo general, remite espontáneamente; la endoscopía de urgencia solo está indicada si los resultados fueran a cambiar la conducta clínica o si se requiriera realizar alguna intervención terapéutica

\section{Clasificación de las Hemorragias}

Según la magnitud y compromiso hemodinámico, se utilizó la clasificación según PALS (American Heart Association) (ver Cuadro 4).

\section{Tratamiento médico}

Hemorragia leve: colocar una SNG, comprobar que no existe sangrado activo, medicar con citoprotector o antiácidos y probar tolerancia oral.

\section{Hemorragia moderada}

- Canalizar vía venosa y administrar líquido cristaloide isotónico a 20 $\mathrm{ml} / \mathrm{Kg}$ en 10-20 minutos hasta estabilización hemodinámica.

- Administrar ranitidina u omeprazol EV.

- Una vez estabilizado hemodinámicamente el paciente, realizar endoscopía alta, para 
información sobre origen del sangrado y pronóstico (ver criterios de Forrest). Con hemorragia activa (Forrets Ia y Ib) se utilizarán métodos endoscópicos para hemostasia.

\section{Hemorragia Severa}

Tratamiento inicial, en la Unidad de cuidados intensivos (UCI):

- Proteger la vía aérea y valorar la necesidad de intubación.

- Mantenimiento de una adecuada oxigenación.

- Canalización de accesos venosos periféricos y catéter venoso central. Para control presión venosa central, respuesta y manejo de fluidos.

- Expansión rápida de la volemia, inicialmente con cristaloide isotónico a $20 \mathrm{ml} / \mathrm{Kg}$ en 5-10 minutos repetidos hasta 3 o 4 veces sino mejora, luego con hemoderivados hasta estabilización hemodinámica.

- Corregir la alteración de la coagulación con la administración de plaquetas y plasma fresco congelado.

- Corrección de los trastornos electrolíticos y metabólicos.

- Colocar sonda vesical y sonda nasogástrica permanente si hay compromiso del sensorio o en el neonato

Una vez estabilizado el paciente, proceder como en la hemorragia moderada

En la Hemorragia variceal: (ver Algoritmo diagnóstico y terapéutico)

A. Tratamiento del episodio agudo:

1.Uso de reductores del flujo esplácnico: Vasopresina, somatostatina, terlipresina y octreótide.

2. Ligadura con bandas o esclerosis con polidocanol al $1 \%$.

3.Sondas balón esofágico (Linton-Nachlas) o de Sengstaken-Blakemore (doble balón).

B. Profilaxis secundaria: La ligadura con bandas (método de elección) o esclerosis. En várices gástricas: Inyección con butil-2 cianoacrilato.

C. Profilaxis primaria: Prevenir o retrasar el primer episodio de sangrado por várices con ligadura con bandas.

D. Profilaxis antibiótica: Debe ser implementado en todo paciente con cirrosis y sangrado del tracto digestivo superior (ver Babeno VI).

\section{ACCIDENTES POR INGESTION DE CUERPOS EXTRAÑOS}

La ingestión de cuerpos extraños (CE) es una de las emergencias más frecuentes de consulta en una guardia pediátrica. Ocurre generalmente dentro del ambiente familiar y bajo el cuidado de los adultos. Por el inicio súbito y la inmediata instalación de síntomas, la ingesta de un CE crea una situación sumamente angustiante para el paciente y sus familiares. La mayoría de los CE (del 80 al 90\%) que llegan al tubo digestivo lo pasan de modo espontáneo sin atascarse, solo el 10 al $-20 \%$ de los casos requiere de algún tipo de intervención, y el 1\% o menos algun procedimiento quirúrgico para su resolución.

(Ver Algoritmo diagnóstico y tratamiento)

- Indicaciones de endoscopia inmediata: Todo cuerpo extraño localizado en esófago, cualquiera sea sus características, se considera una emergencia por lo cual debe ser extraído antes de la 24 hs de ingestión por el alto riesgo a la lesión por decúbito y posterior perforación.

- Endoscopia diferida: cuando el paciente no presenta un ayuno adecuado y carece de signos y síntomas que obligan a realizar un procedimiento de urgencia, en este caso el estudio se realizará cuando el paciente mantenga un ayuno adecuado.

- Las pilas constituyen un capítulo aparte, ya que el alto riesgo de complicaciones que puede traer su estacionamiento lleva a su extracción en tiempos más cortos que los otros cuerpos extraños; se recomienda su extracción ante el diagnóstico.

- Conducta expectante: En niños y adultos con ingesta de $\mathrm{CE}$ romos pequeños y cuando el CE haya pasado el píloro. Se controlará progresión del mismo con radiografías seriadas y su eliminación por vía natural.

- Cuando el objeto es mayor de $20 \mathrm{~mm}$ de diámetro o mayor de $5 \mathrm{~cm}$ de longitud es dificultoso que puedan pasar el píloro en un niño pequeño en forma espontánea, por lo cual cualquiera sea la característica del mismo se recomienda su extracción endoscópica. Se evaluará la extracción quirúrgica si el objeto es corto punzante/ pila que no progrese y presente síntomas.

(Ver Tabla1. Indicaciones de extracción de cuerpos extraños y Figura 1. Algoritmo diagnóstico y manejo de $C E)$. 


\section{ACCIDENTES POR INGESTIÒN DE CÀUSTICOS}

La ingesta de productos cáusticos sigue siendo un grave problema médico social, ya que la prevención de estos accidentes es esencial para evitar lesiones del tubo digestivo que generan una morbimortalidad importante a corto y a largo plazo. Por un lado, la mayoría de las ingestas de productos cáusticos se producen en forma accidental en el hogar. El grupo de mayor riesgo son los niños menores de 5 años, con un pico de máxima incidencia alrededor de los 2 años, cuando logran autonomía para la deambulación, pero todavía no pueden reconocer situaciones y objetos de peligro. Por otro lado, se pueden producir ingestas voluntarias con intento autolítico en adolescentes, con ingesta de mayor cantidad del producto y, por consecuencia, mayor riesgo de sufrir lesiones graves del tracto digestivo.

\section{Tipos de cáusticos}

- Ácidos: clorhídrico, sulfúrico, fosfórico, acético o nítrico.

Los ácidos actúan con un $\mathrm{pH}$ menor a 4. Producen necrosis por licuefacción, desnaturalización de las proteínas, saponificación de las grasas $\mathrm{y}$ trombosis vascular

- Alacalis: lejía, amoniaco, desincrustantes y detergentes.

Actúan con un pH igual o mayor a 12.

Producen necrosis por coagulación de proteínas, perdida de agua y formación de escara.

La toxicidad del cáustico se relaciona con: el tipo del producto ingerido (álcali o ácido), las propiedades físicas (sólido o líquido), químicas (concentración y $\mathrm{pH}$ ) y el volumen ingerido, influyendo en la gravedad de las lesiones.

Debe realizarse una evaluación del paciente con buen interrogatorio, examen clínico y endoscopia si lo requiriera.

Conducta según evaluación: (ver Figura1. Algoritmo diagnóstico y terapéutico)

- Alta del paciente: Ingestión dudosa, sin signos clínicos o aquellos con ingestión de lejía diluida. Endoscopia no indicada. Control ambulatorio con signos de alarma.
- Observación del paciente: Ingesta segura de cáustico, estable con o sin síntomas y / o lesiones bucales. Se indica endoscopia, ayuno, protección gástrica con inhibidor de la bomba de protones (IBP).

a) Endoscopia sin lesiones: Probar tolerancia oral con líquidos claros. Programar el alta con IBP y control con gastroenterología.

b) Endoscopia con lesiones: Internación del paciente.

- Lesión esofágica Grado I y IIa: internación en sala, líquidos claros, IBP, analgésicos. Alta cuando se resuelven los síntomas y adecuada tolerancia oral.

- Lesión esofágica Grado IIb y III: internación en UCI, reposo gástrico, medidas de soporte vital hemodinámicas, respiratorias, IBP, analgésicos, corticoides y antibióticos (ver Clasificación Endoscópica de Zargar).

Evaluar la necesidad de una nutrición parenteral cuando la vía enteral no es posible o está contraindicada.

- Control de la evolución:

- Esofagograma después de los 30 días, posterior a la ingesta (para evaluar presencia de estenosis)

- Endoscopia de control: Si no presenta complicaciones no sería necesaria.

- Tratamiento quirúrgico

La cirugía urgente está indicada en sospecha perforación esofágica o gástrica. En quemaduras extensas de esófago, debe considerarse la posibilidad de una gastrostomía.

\section{CONCLUSIONES}

Se ha confeccionado este consenso con la colaboración de la mayoría de los referentes de la especialidad en gastroenterología y endoscopia pediátrica del país. En cada una de ellas expone una revisión actualizada, una guía de diagnóstico, algoritmos, terapéutica recomendada y medidas preventivas que se podrían tomar para evitar algunos de estos accidentes y disminuir la morbilidad de las mismas.

Nota: Los cuadros, algoritmos, figuras y tablas se encuentran disponibles en la versión completa en formato electrónico. 